\title{
Reliability of Extended Kalman Filtering Technic on Vehicle Mass Estimation
}

\author{
Mert Büyükköprü ${ }^{1 *(D)}$, Erdem UZUNSOY ${ }^{2}$ \\ 1* Groupe Renault, Bursa, Turkey \\ 2 Bursa Technical University, Mechanical Engineering Department, 16310, Bursa, Turkey
}

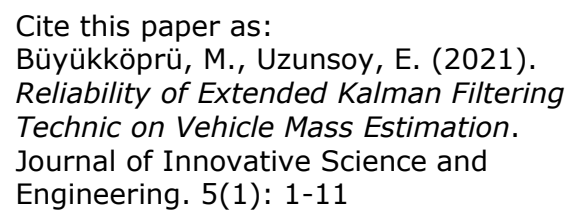

The works published in Journal of Innovative Science and Engineering (JISE) are licensed under a Creative Commons Attribution-NonCommercial 4.0 International License.

\begin{abstract}
In this paper, vehicle mass estimation problem was researched by the Kalman Filtering process to investigate the effectiveness of Extended Kalman Filter technique on mass estimation, which has widely been studied on the related literature with all the successful results. For the purpose, a longitudinal vehicle dynamics model was developed, and the equations were transformed into the well-known state space form. Nonlinear problem in its nature was discretised and linearized by using Euler method. In addition, road slope was calculated by a road slope inclinometer logic. IPG CarMaker ${ }^{\circledR}$ was utilized to overcome the nonlinearities, design the test environment and the conditions, as it was observed that the simulation results were not met the expectations with the logged vehicle physical test data. Several test scenarios were run to simulate level and wavy road with different vehicle speeds. The results showed that the Extended Kalman Filter could provide a reasonable convergence when the initial values were correctly selected, no nonlinear issue occurred and certain tuning parameters were defined with stable boundary conditions for mass estimation.
\end{abstract}

Keywords: Extended Kalman Filter, Mass Estimation, Vehicle Longitudinal Dynamics 


\section{Introduction}

Vehicle mass is a significant parameter in the dynamic response, control and energy management of a vehicle. Force and moment generation potential of pneumatic tyre-road interface is directly affected by the dynamic load transfer and changing mass due to the different loadings. Nowadays, as many modern vehicles are equipped with many different sensors such as vehicle speed and accelerometers, signals of the sensors are already available in a common communication network, and therefore, automotive researchers exert effort to utilize the additional benefits of those sensors. Utilising on-board sensors instead of using external is one of the economic reasons for the researchers. As a benefit of the approach, considering the positive effects on the results of vehicle dynamics models, online mass and road slope estimation algorithms, which are actually some sort of virtual sensors, have extensively been studied by the researchers, in addition to the conventional dynamic load transfer calculation, in the literature. When the forces and moments such as wheel torque, resistance force due to road slope, air resistance, rolling resistance of tyres, and some measured vehicle acceleration parameters are known, an unknown parameter of the vehicle system can be estimated.

Lingman et al. [1] focused on Road slope and mass estimation using Kalman Filter (KF). When the vehicle mass was known they studied to estimate road slope by using speed and some specific force measurements. In the study, different estimators were developed for mass and slope, separately, and the slope estimation and propulsive forces were calculated based on engine fuel injection amount. The brake force was not considered in the model due to the nonlinearity of the friction. When there was an active braking, related data was omitted during the simulation. It was found that Extended Kalman Filter (EKF) was feasible for the purpose. L. Imsland et al. [2] developed a nonlinear observer for vehicle velocity with friction and road bank angle adaptation with nonlinear filter. They used 3 Degree of Freedom (DOF) vehicle model including the road bank angle. Results were as good as with the EKF. Vahidi et al. [3] studied recursive least squares (RLS) with multiple forgetting factor for online estimation of vehicle mass and road grade for heavy duty vehicles. A forgetting factor for each parameter to weight the previous and present data to determine the next. A commercial test vehicle was used to obtain the data and the estimated mass error was found at around \%5. WraggeMorley et al. [4] focused on gradient and mass estimation from CAN based data for a light passenger car. They followed two different steps to increase the accuracy of estimated mass output. In the first step, the period of convergence was calculated and the inappropriate outputs were rejected. Then, in the second step, KF was used to provide a smooth output. Road slope was calculated via longitudinal accelerometer. Smooth results were achieved in short settling time at around 50s and with approximately $10 \%$ error. Kidambi et al. [5] assessed the accuracy and performance of four estimation methods, which were RLS, EKF, Dynamic grade observer and parallel mass and grade estimation using a longitudinal accelerometer, on road slope and vehicle mass estimation. Drive torque was measured at the front-left and front-right shaft by strain-gauges instead of the calculation of tractive force. They found that the estimations of RLS and EKF produced the error under 5\%, with the realistic initial values. It was observed that when initial values are not accurate enough, algorithm converges with a residual error. Huh et al. [6] 
developed a unified estimation algorithm especially for adaptive roll control which had three different mass estimators using vehicle longitudinal, lateral and vertical dynamics. RLS, KF and dual recursive least squares techniques were used to determine the vehicle sprung mass, in the longitudinal, lateral and vertical dynamics estimators, respectively. Wenzel et al. [7] studied Dual Extended Kalman filter technique on a four-wheel vehicle model to estimate vehicle dynamics states such as longitudinal velocity, lateral velocity, yaw rate and roll angle and the parameters like mass etc. The key point of the approach was to switch off one of the EKF when the other one provided a good estimation to prevent the over processing.

Pence et al. [8] estimated the sprung mass of an off-road vehicle using RLS method. By considering the sprung and unsprung mass accelerations in a quarter car model, the mass was estimated within 8\% error, under 100s. Bae et al. [9] utilized GPS signals to estimate road gradient. Then, estimated road gradient was used to determine the wheel torque, to be used in the estimation of the vehicle mass via a recursive algorithm. Results were within $2 \%$ of the real values. Raffone [10] designed an observer using the KF method to calculate the road slope based accelerometer measurement on vehicle, and estimated the vehicle mass using RLS method with forgetting factor. Experimental test results showed that the mass estimator converged after around $150 \mathrm{~s}$ for three urban road scenarios. Holm [11] developed EKF to determine heavy commercial vehicle mass using road slope information provided by on-board sensors. Vehicle transmission and final drive inertias were included in the longitudinal model as parts of the inertial resistance. To eliminate the convergence problems and decrease the estimation error, a logic was developed disregarding braking and caring the experienced threshold values for vehicle speed and engine torque. The physical experiments showed that EKF could estimate the mass, correctly, after around 50s. However, due to the mass selection algorithm, total cycle time was 300 s.

In this study, a longitudinal vehicle dynamics model was developed and Newtonian model equations were transformed into the well-known state space form for the Kalman Filtering process to investigate the effectiveness of Kalman Filter technique on mass estimation, which has widely been studied on the related literature with the successful results. Firstly, Simulation was done offline by logged vehicle physical test data. Secondly, IPG CarMaker ${ }^{\circledR}$ was utilized to overcome the nonlinearities in the real data and to design the test conditions and environment. The contribution of this study to the existing literature is simply to present a full projection of the method on general vehicle mass estimation, rather than focusing on the very limited and in fact conditionally successful simulation results as presented in the literature, generally. It was seen that the KF only converges when the initial values were correctly selected, and the vehicular inputs causing the nonlinearities were eliminated for mass estimation.

\section{Vehicle Longitudinal Dynamics Model}

Road slope was calculated by an inclinometer logic, which was developed by Renault. Inputs were wheel speeds, vehicle speed, calculated road slope and powertrain wheel torque. Due to the nature of conventional Kalman Filtering technic, it is normally applicable only to the systems represented by the linear equations. The problem which was considered in this study, on the other hand, includes nonlinearities and an alternative solution of this kind of problems is EKF. It is a modified 
version of KF, and nonlinear problems are, in fact, linearized around the working point. Road and vehicle dynamics data were collected from IPG CarMaker ${ }^{\circledR}$, which simply represents a virtual vehicle test capabilities to develop and test the systems [12] in a highly controllable environment [13], for the validation of vehicle longitudinal dynamics model developed for this study. On the basis of this model, an estimation algorithm was developed, and the simulation results were discussed based on some the scenarios.

The Figure 1 shows the free body diagram of a vehicle on a sloped road. In the figure, $F_{-} g r a d e, F_{-} r, F_{-} a e r o$, $F_{-} T q, T_{-} q, r, F_{-} b$ represent gradient resistance, rolling resistance of tyres, air resistance, tractive force, wheel torque, tyre rolling radius, and braking force, respectively. Powertrain wheel torque calculation can simply be expressed as in equation (1) without slip between the road and tyre interface.

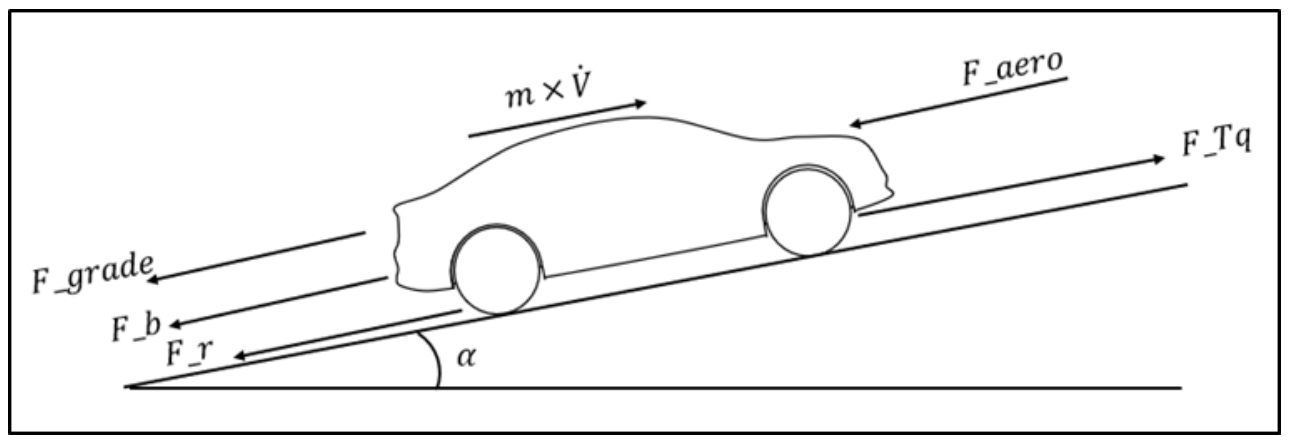

Figure 1. Forces applied to a vehicle on a sloped road

$$
\begin{aligned}
& F_{T q}=T q / r \\
& F_{T q}-F_{r}-F_{\text {aero }}-F_{\text {grade }}=m \dot{V} \\
& V=\frac{T_{q}}{m r}-g f \cos (\alpha)-\frac{1}{2 m} \rho A C_{D} V^{2}-g \sin (\alpha)
\end{aligned}
$$

The condition for the motion is given in equation (2). By replacing the equivalences of the forces in the equation, vehicle speed can be derived from the second order expression in equation (3). The expressions $g, f, C_{D}, \rho, A, T q, r, V$, refer to gravity, rolling resistance coefficient, air drag coefficient, air density, vehicle frontal area, wheel torque, rolling radius and vehicle speed, respectively. As another important quantity of a longitudinal vehicle model, braking force, which is developed by the friction of braking pads, is inherently nonlinear and the tyre-road friction cannot be measured or calculated, exactly, by the classical mechanics. Additionally, the tyres naturally slips and it deteriorates the tractive force calculation during braking. As a result, braking force was not taken into account in the equation (2) [14].

Kalman filter is a linear estimation algorithm for linear systems and it is represented in state space form [15]. $V, \mathrm{~m}, \alpha$ are the velocity, total mass of vehicle and road slope, respectively, and they are the states of the system shown in equation (4). In the equation, velocity and road slope are measured and mass is an estimated quantity. 


$$
x=\left[\begin{array}{c}
V \\
m \\
\alpha
\end{array}\right]
$$

As Kalman filter is a technique for linear problems, and EKF is used for nonlinear equations, thus, discretization with Euler method can be applied as in equation 5.

$$
\left\{\begin{array}{c}
V_{k}=V_{k-1}+\Delta t \times\left(\frac{T q}{m \times r}-g \times f \times \cos (\alpha)-\frac{1}{2 \times m} \times C_{D} \times A \times \rho \times V^{2}-g \times \sin (\alpha)\right) \\
m_{k}=m_{k-1} \\
\alpha_{k}=\alpha_{k-1}
\end{array}\right.
$$

For small angles of $\alpha, \sin (\alpha) \cong \alpha \& \cos (\alpha) \cong 1$ are assumed and then, equation 3 can be simplified into the form as in equation 6.

$$
\dot{V}=\frac{T q}{m \times r}-g \times f-\frac{1}{2 \times m} \times C_{D} \times A \times \rho \times V^{2}-g \times \alpha
$$

After the linearization of the equations, fundamental matrix, which is known as Jacobian matrix, is designed and the columns of the matrix are derived with respect to $v, m$, and $\alpha$ as they are shown in equations 7 and 8 .

$$
\begin{gathered}
F=\left[\begin{array}{lll}
\frac{d f_{1}}{d v} & \frac{d f_{2}}{d m} & \frac{d f_{3}}{d \alpha} \\
\frac{d f_{1}}{d v} & \frac{d f_{2}}{d m} & \frac{d f_{3}}{d \alpha} \\
\frac{d f_{1}}{d v} & \frac{d f_{2}}{d m} & \frac{d f_{3}}{d \alpha}
\end{array}\right] \\
F=\left[\begin{array}{ccc}
1-\frac{C_{D} \times A \times \rho \times V}{m} \times \Delta t & \frac{C_{D} \times A \times \rho \times V^{2}-2 \times T q}{2 \times m^{2} \times r} \times \Delta t & -g \times \Delta t \\
0 & 0 & 0 \\
0 & 0 & 0
\end{array}\right]
\end{gathered}
$$

Measurements represented by ' $y$ ' include vehicle speed and road slope and $\mathrm{H}$ is the measurement and $w_{z}$ is the measurement noise, incorporated in R matrix, in equation 9.

$$
y=H x+w_{z}
$$

Measurement variance matrix is represented in equation 10 and the dimension of $\mathrm{R}$ matrix depends on the number of the measurements. In this study, vehicle speed and road slope were measured and some proper sensor measurement errors were selected. Measurement errors may be selected different than their theoretical errors, while process noise and 
measurement error matrix are also used as the tuning knob of the system. Measurement covariance was set to zero, hence, road slope and vehicle speed were taken as independent measurements.

$$
R=\left[\begin{array}{cc}
\sigma_{V}^{2} & 0 \\
0 & \sigma_{\alpha}^{2}
\end{array}\right]
$$

Variance and covariances of each state variable are shown in process noise matrix, in equation 11;

$$
Q=\left[\begin{array}{ccc}
\sigma_{V}^{2} & 0 & 0 \\
0 & \sigma_{m}^{2} & 0 \\
0 & 0 & \sigma_{\alpha}^{2}
\end{array}\right]
$$

Inclinometer used in this study is an algorithm that uses longitudinal accelerometer measurements, $a_{x}$, and vehicle longitudinal velocity, $V_{x}$, at driven axle wheels. Basically, on a flat and level road surface, vectors of $a_{x}$ and $V_{x}$ point the same direction, otherwise vehicle is tilted due to the road grade or pitch, and the measured $a_{x}$ component includes the road grade component of gravity and vehicle longitudinal acceleration as presented in equation 12 . This equation simply produces the road slope given in equation 13 [16].

$$
\begin{aligned}
& a_{x}=\dot{V}_{x}+g \cdot \sin \alpha \\
& \alpha=\arcsin \left(\left(a_{x}-\dot{V}_{x}\right) / g\right)
\end{aligned}
$$

In the first stage of the study, several data were collected from the vehicle tests to perform offline calculations in MATLAB/Simulink ${ }^{\circledR}$ to observe the vehicle mass and road grade. Due to the nonlinearities such as variable slope of the road, various manoeuvres and the gear shifting necessities caused by the traffic conditions, the results were incomprehensible. Therefore, it was decided to provide road and vehicle data in a simulation environment, which is capable of to design the vehicle and road conditions, flexibly, allowing to reduce the nonlinearities. IPG CarMaker ${ }^{\circledR}$ is one of the powerful and well-known tools to design and represent the vehicle, road, driving conditions and the test environment. Thus, three scenarios and driving conditions were designed by using the capabilities of the software. Table 1 represents the simulation scenarios.

Table 1. Simulation scenarios

\begin{tabular}{llllll}
\hline & & & & \multicolumn{2}{c}{ Braking } \\
Scenario & Road Condition & Driving Conditions & Grade & Speed & Status \\
\hline 1 & Level road with small changes & IPG Driver Model & $-/+1^{\circ}$ & $120 \mathrm{kph}$ & None \\
2 & Wavy road with grade change & IPG Driver Model & $-/+5.5^{\circ}$ & $100 \mathrm{kph}$ & Braking \\
3 & Wavy road grade sharp grade change & IPG Driver Model & $-/+5.5^{\circ}$ & $80 \mathrm{kph}$ & Braking \\
\hline
\end{tabular}


In the first scenario, neither braking nor gear shifting were applied. Vehicle speed was set to around 120kph (Figure 2) on a level road (Figure 3), which was to simulate a highway and small engine torques were applied to keep the vehicle at a constant speed. For a good estimation, initial mass value was set as close as the real value. Therefore, $1800 \mathrm{~kg}$, which 60 $\mathrm{kg}$ is less than the real mass, was chosen.

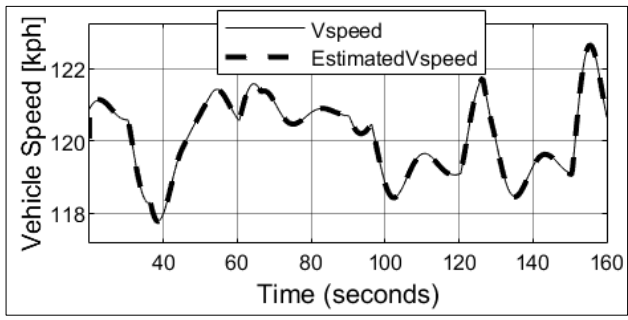

Figure 2. Scenario no:1 speed profile

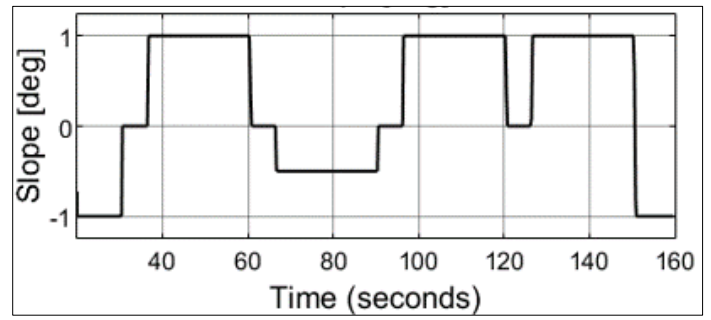

Figure 3. Scenario no:1 road height profile

The second scenario was designed to observe the severe braking effects on the estimator. Therefore, the vehicle was speeded up to $100 \mathrm{kph}$ and the maximum braking force was applied to a full stop. The same manoeuvres were repeated three times for the duration of 100s (Figure 4). Figure 5 shows the road height profile while Figure 6 shows the braking status during the simulation.

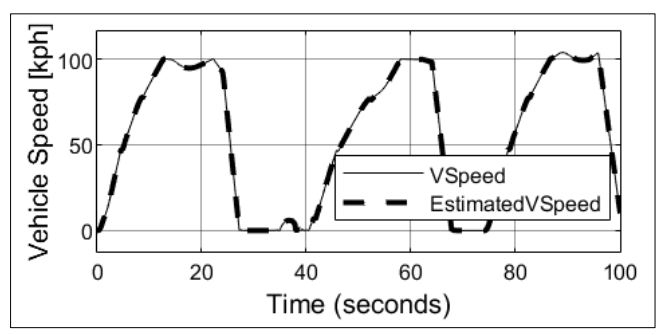

Figure 4. Scenario no:2 speed profile

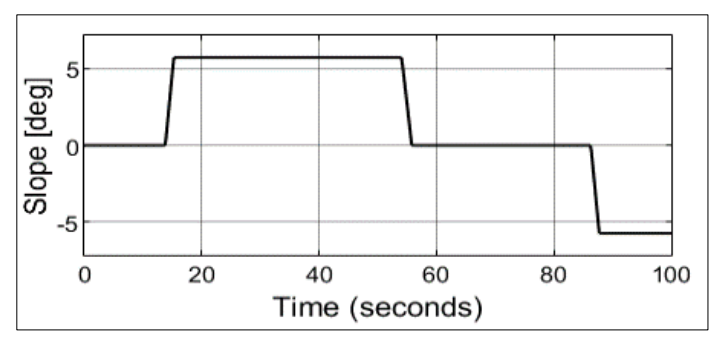

Figure 5. Scenario no:2 road height profile

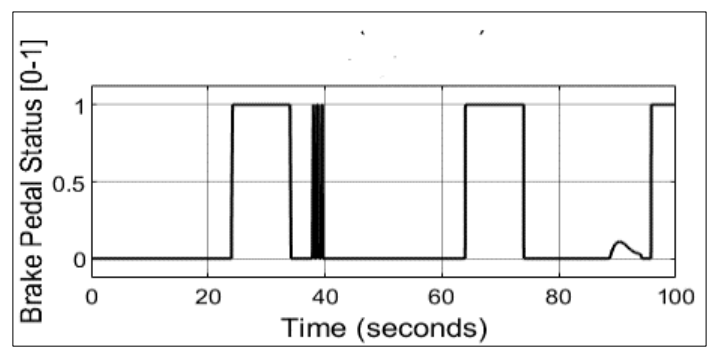

Figure 6. Scenario no:2 braking status

In the third scenario, vehicle was speeded up to $80 \mathrm{kph}$ with the road slope variation of $-/+6^{\circ}$ during the $350 \mathrm{~s}$ (Figure 7 and 8). While the vehicle was going downhill, IPG Driver applied small brakes to keep the vehicle speed constant at around 50,120 and 260 seconds of the simulation as seen in the Figure 9. 


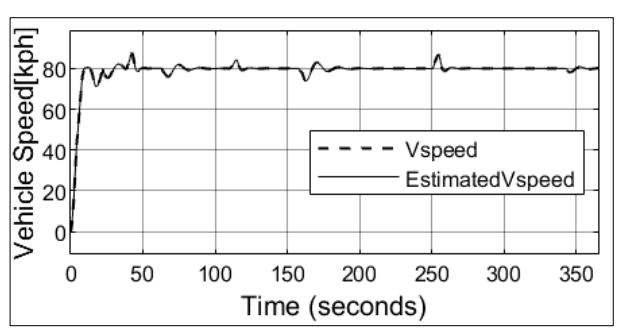

Figure 7. Scenario no:3 speed profile

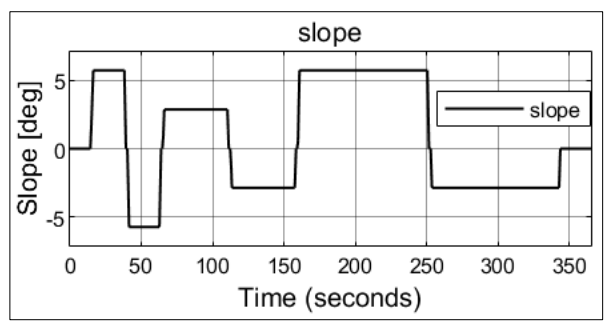

Figure 8. Scenario no:3 road height profile

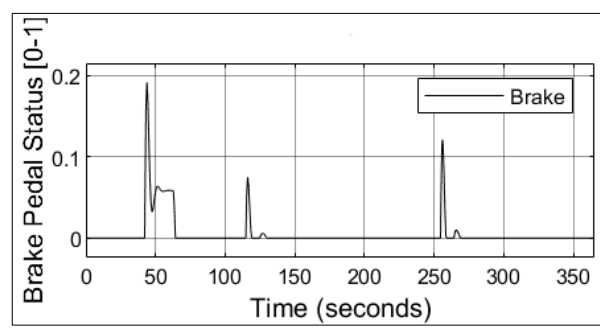

Figure 9. Scenario no:3 braking status

\section{Results and Discussion}

Three scenarios were studied to reveal the effects of the gear shifting and longitudinal forces applied on the vehicle for the purpose of providing an acceptable mass estimator. Braking and gear shifting were important to observe their negative effects on the estimation.

In the first scenario, vehicle speed was set to around $120 \mathrm{kph}$ on a level road, representing a highway, without any brake and gear shifting input. Small engine torques were applied to keep the vehicle speed constant. For the accuracy of the estimation, initial mass value was set close to the real value as $57 \mathrm{~kg}$ less than the real mass. The results showed that the estimated mass was converged to the real value at around 150s (Figure 10), on the other hand, it was seen that the estimation was quite sensitive to the wheel torque changes after 150s (Figure11). However, while the vehicle was being driven downhill, the lower tractive force demand was affected the estimation. Thus, the estimated mass diverged from the real value. With an appropriate initial value and without any disturbance, estimator error was less than $\% 5$.

In the second scenario, vehicle was accelerated up to $100 \mathrm{kph}$ by severe wheel torque application and the data included the gear shifting and a severe braking for a full stop. The first braking was applied at around 40s and afterwards, it was repeated two times at 120s and 260s. As a result, estimated mass increased, significantly (Figure 12 and 13).

In the third scenario, vehicle was started to move from stand still, and the speed was increased to 80kph. After reaching the target speed, no gear shifting was applied. Wheel torque was smooth. When the vehicle was in downhill, three braking inputs were applied by the cruise control of IPG driver at 50s,120s and 260s to keep the speed constant. The three braking inputs were increased the estimated mass (Figure 14 and 15). 


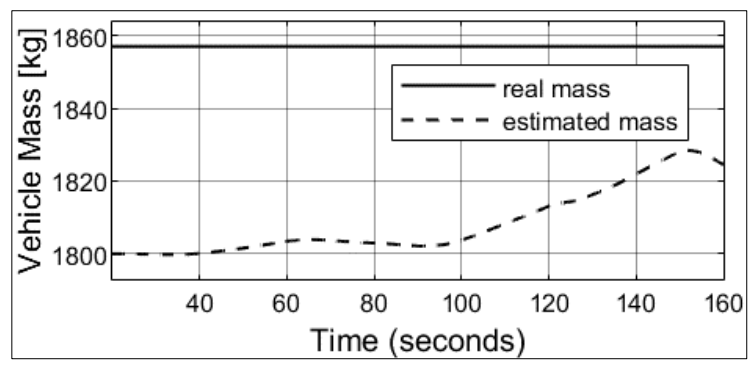

Figure10. Scenario no:1 estimated with respect to real mass.

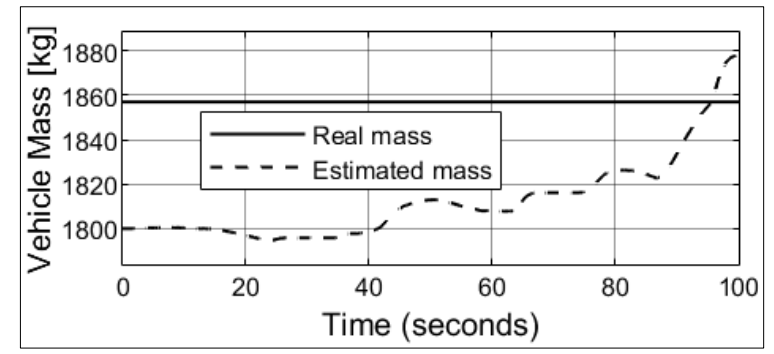

Figure 212. Scenario no: 2 with respect to real mass

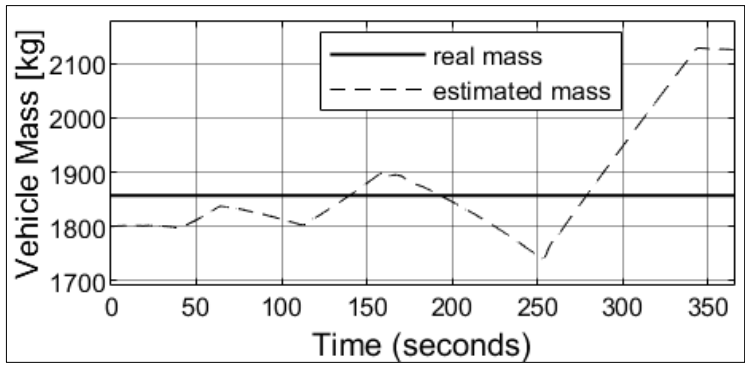

Figure14. Scenario no:3 with respect to real mass

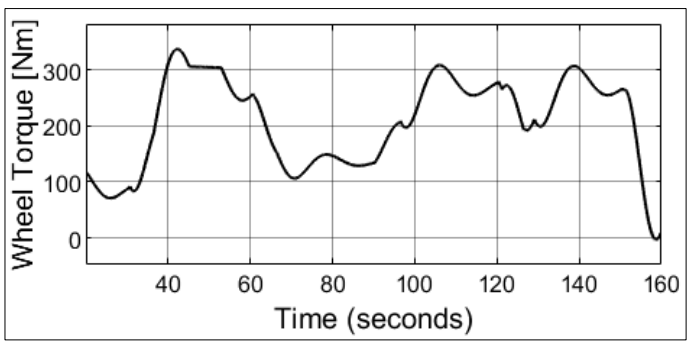

Figure11. Scenario no:1 Wheel torque

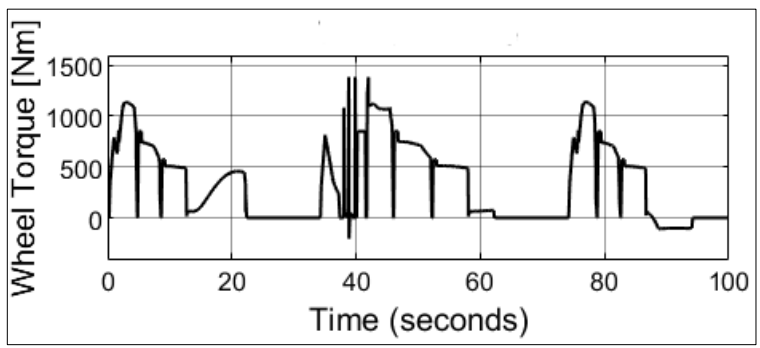

Figure13. Scenario no:2 Wheel torque

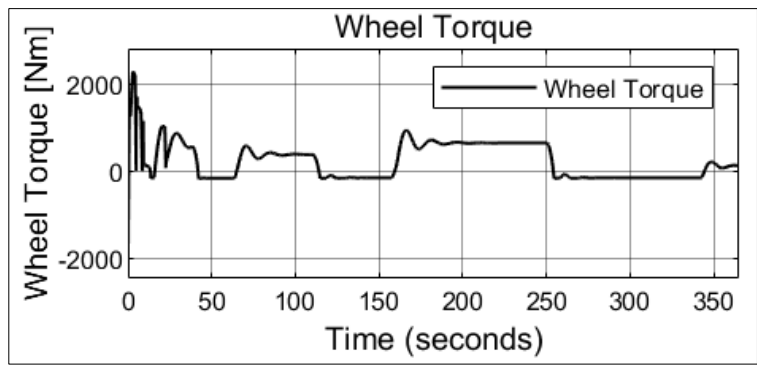

Figure15. Scenario no:3 Wheel torque

It's known that appropriate initial value is strongly needed for accurate estimation and proper filter tuning parameters. The first scenario's results showed that EKF works fine with appropriate initial value and the smooth data, which includes braking or gear shifting for accurate estimation within acceptable error range. The second scenario's results showed that braking force was a disturbance for the estimation. To eliminate the over or under estimation, either the braking should be included in the equations or the selected data must not include any braking input. The third scenario's results underlined that even a small amount of braking input was existed, EKF systematically overestimates and causes error, which was around $13 \%$ in the case of this study. As a result of this, it is possible to have a conclusion that either all the disturbances should be taken into consideration or compensated by additional algorithms.

\section{Conclusion}

Longitudinal dynamics equations were derived under the assumptions such as, tyre was not sliding and road adhesion and tyre dynamic radius were constant and tractive force at wheel was accurate. However, the total error of the model was increased by these assumptions. It is known that the tractive force provided by the tyre-road interface is calculated based 
on engine map and the accuracy decreases due to the alternator and air conditioner engagement. Due to physical experiment bottlenecks, offline studies were carried out by IPG CarMaker ${ }^{\circledR}$, which has a highly controllable environment. Based on the worst case test results, $13 \%$ error was observed during the tests with the data provided by IPG CarMaker ${ }^{\circledR}$, which is a value, nearly half of the useful load of a typical passenger car. On the contrary of the presented results in the literature, it is difficult to have a valid outcome. EKF could only give the reasonable outcomes under some certain and limited conditions, as depended on the correct initial information and some certain tuning parameters defined for the stable boundary conditions. Additionally, estimated mass output should be validated, carefully. Therefore, to validate the results, estimated value can be re-estimated more than once during the calculation. So, it could be inferred that to provide the correct initial value to the filter and to validate the estimation results, alternative out-source variables may be needed for the corrections. Nevertheless, estimation can be more robust for future vehicles, which will have more accurate tractive force output of electric motors with more sensors on-board.

\section{Acknowledgements}

This study was a part of the research, financially supported by The Scientific and Technical Research Council of Turkey (TUBITAK-TEYDEB) with grant number of 3170303.

\section{References}

[1] Lingman, P., Schmidtbauer, B. (2002). Road Slope and Vehicle Mass Estimation Using Kalman Filtering. International Journal of Vehicle Mechanics and Mobility, 37 (1):12-23.

[2] Imsland, L., Grip, H. F.,Johansen A.T.,Fossen T. I., Kalkkuhl, C. J., Suissa, A. (2007). Nonlinear Observer For Vehicle Velocity With Friction And Road Bank Angle Adaptation With An Extended Kalman Filter. SAE International World congress Detroit, Michigan April 16-19 2007.

[3] Vahidi, A., Stefanopoulou, A., Peng, H., (2005). Recursive least squares with forgetting for online estimation of vehicle mass and road grade: theory and experiments. Vehicle System Dynamics, 43(1): 31-35.

[4] Wragge-Morley, R., Hermann, G., Barber, P., Burgess, S., (2015). Gradient and Mass Estimation from CAN Based Data for a light passenger Car. SAE International J. Passenger Cars-Electronic Electric Systems, 8 (1):137-145.

[5] Kidambi, N., Harne, R.L., Fuji, Y., Pietron, G. M., Wang, K.W., (2014). Methods in Vehicle Mass and Road Grade Estimation. SAE International J. Passenger Cars -Mechanical Systems, 7(3): 981-991.

[6] Huh, K., Lim, S., Jung, J., Hong, D., Han, Han, K., Jo H. Y., Yun, J. M., (2007). Vehicle Mass Estimator for Adaptive Roll Stability Control. SAE International World congress Detroit, Michigan ,16-19 April 2007.

[7] Wenzel, T.A., Burnham, K. J., Blundell, M. V., Williams, R.A., (2007). Dual extended Kalman filter for vehicle state and parameter estimation. Vehicle System Dynamics, 44(2): 153-171.

[8] Pence., B. L., Fathy K. H., Stein, J. L., (2009). Sprung Mass Estimation for Off-Road Vehicles via BaseExcitation Suspension Dynamics and Recursive Least Squares. American Control Conference, Hyatt Regency Riverfront, St. Louis, MO, USA. June 10-12, 2009.

[9] Bae, H. S., Gerdes J. C., (2003). Parameter Estimation And Command Modification For Longitudinal Control Of Heavy Vehicles. California PATH Research Report UCB-ITS-PRR-2003-16. ISSN: 1055-1425. 
[10] Raffone, R., (2013). Road Slope and Vehicle Mass Estimation for Light Commercial Vehicle using linear Kalman filter and RLS with forgetting factor integrated approach. 16th International Conference on Information Fusion Istanbul, Turkey, July 9-12, 2013.

[11] Holm, E.J. (2011). Vehicle Road Grade And Mass Estimation Using Kalman Filter. MSc. Thesis, Linköping University, Linköping.

[12] URL 1: Ipg Automotive (2019). CarMaker: Virtual testing of automobiles and light-duty vehicles. https://ipgautomotive.com/products-services/simulation-software/carmaker/. [Accessed: 13 December 2019.]

[13] Karoshi., P., Ager, M., Schabauer, M., and Lex, C., (2017). Robust and Numerically Efficient Estimation of Vehicle Mass and Road Grade. Advanced Microsystems for Automotive Applications 2017. 26 September, Berlin, Germany. pp. 87-100.

[14] Lundin, B., Olsson, A., (2012). Estimation of Vehicle Mass Using and Extended Kalman Filter. MSc. Thesis. Chalmers University of Technology, Gothenburg, Sweden.

[15] Roger R. L., (2018). "Kalman and Bayesian Filters in Python" https://www.academia.edu/37533812/Kalman_and_Bayesian_Filters_in_Python [Accessed: 14 December 2019]

[16] Ohnishi, H., Ishii, J., Kayano M., Katayama, H., (2000), “A Study On Road Slope Estimation For Automatic Transmission Control”, Society of Automotive Engineers of Japan, 21(2): 235-240. 